Discrete Comput Geom 27:353-375 (2002)

DOI: $10.1007 / \mathrm{s} 00454-001-0078-2$

Geometry

\title{
Closed-Form Expressions for Uniform Polyhedra and Their Duals
}

\author{
Peter W. Messer \\ 4315 West Riverlake Drive, \\ Mequon, WI 53092-4856, USA \\ pmesser@wi.rr.com
}

\begin{abstract}
A uniform polyhedron has equivalent vertices and regular polygonal faces. An established set of 77 Wythoff symbols effectively describes the dynamic kaleidoscopic constructions of uniform polyhedra. The main combinatorial and metrical quantities of uniform polyhedra and their duals are presented as closed-form expressions derived from the Wythoff symbols.
\end{abstract}

\section{Introduction}

The standard definition of a uniform polyhedron is a polyhedron that has regular faces and a symmetry group that is transitive on the vertices. Such a finite figure consists of one or more kinds of faces that are regular plane polygons which meet two to a side (edge) and which are arranged alike around every vertex. With the restriction that no subset of faces has these properties, we exclude a compound of two or more uniform polyhedra.

The 77 kinds of uniform polyhedra are separated historically and naturally into the 5 Platonic solids (convex regular polyhedra), the 13 Archimedean solids (convex semiregular polyhedra), the 4 star polyhedra of Kepler-Poinsot (non-convex regular polyhedra), the 53 non-regular star polyhedra, and the 2 infinite families of uniform prisms and antiprisms.

The complete set of Wythoff symbols is an established system of notation that effectively describes the dynamic kaleidoscopic construction for each uniform polyhedron [7]. The main combinatorial and metrical quantities of uniform polyhedra are presented here as closed-form expressions derived from the Wythoff symbols. This direct approach differs from previous algorithms which involve numeric iterations [14], [21]. A vertex in Cartesian coordinates is located first by one of two different methods (snub case versus 
non-snub case) after which formulas apply to all uniform polyhedra. The known quantities of a uniform polyhedron are then used for deriving expressions for the corresponding dual polyhedron.

The concepts, terminology, and many of the symbol conventions used in this paper follow primarily [5] and [7]. Important relations known to be in the literature are referenced as such. To simplify the relationships, all derived metrical quantities are based on uniform polyhedra of edge-length 2 except where indicated. The formulas, most of which are new, are presented in ways that adapt well to symbolic computer programming. In this regard, the author has written a program using Mathematica (Wolfram Research, Inc.) which renders the labeled vertex figure and returns the important set of exact metrical quantities for the uniform polyhedron given by its Wythoff symbol. Other available Mathematica programs render the complete uniform polyhedron or its dual [21], [38].

\section{A Brief Review of Wythoff's Kaleidoscopic Constructions and Symbols}

Except for one non-Wythoffian case, uniform polyhedra can be generated by Wythoff's kaleidoscopic method of construction. In this construction an initial vertex inside a special spherical triangle $P Q R$ is mapped to all the other vertices by repeated reflections across the three planar sides of this triangle. Likewise in such a trihedral kaleidoscope, $P Q R$ and all its images must cover the sphere an integral number of times which is referred to as the density $d$ of $P Q R$. The density $d \geq 1$ is dependent on the choice of angles $\pi / p, \pi / q, \pi / r$ at $P, Q, R$, respectively, where $p, q, r$ are reduced rational numbers greater than one. Such a spherical triangle is called a Schwarz triangle, conveniently denoted $(p q r)$. Except for the infinite dihedral family of $\left(\begin{array}{ll}p & 2\end{array}\right)$ for $p=2,3,4, \ldots$ there are only 44 kinds of Schwarz triangles [5], [7], [18]. It has been shown that the numerators of $p, q, r$ are limited to 2, 3, 4, 5 (4 and 5 cannot occur together) and so the nine choices for rational numbers are $2,3, \frac{3}{2}, 4, \frac{4}{3}, 5, \frac{5}{2}, \frac{5}{3}, \frac{5}{4}$.

Wythoff's kaleidoscopic constructions fall into one of the four polyhedral symmetry groups: dihedral, tetrahedral, octahedral, or icosahedral. The latter three symmetry groups correspond to the largest numerators 3,4 , and 5, respectively.

There are only three Schwarz triangles that have $d=1$. They are the so-called Möbius triangles (2 33 ), (2 3 4), (2 3 5), spherical triangles which are the fundamental domains for the tetrahedral, octahedral, icosahedral symmetry groups, respectively. The sphere is covered by $g$ Möbius triangles where $g$ is the order of the full symmetry group, that is, $g=24,48$, or 120 respectively for tetrahedral, octahedral, or icosahedral symmetry. It is known [7] that ( $p q r$ ) consists of $d$ Möbius triangles of the same symmetry kind where $d=(g / 4)(1 / p+1 / q+1 / r-1)$.

In the course of Wythoff's kaleidoscopic construction we find that edges join vertices reflected across the sides shared by adjacent Schwarz triangles. However, in the case of snub polyhedra, vertices are generated by an even number of reflections and edges correspond to vertex reflections across the sides of two sequentially adjacent Schwarz triangles [7].

The Wythoff symbol appears simply as a left-to-right sequence of four elements consisting of the three rational numbers $p, q, r$ introduced above and one vertical bar " " 
which separates the numbers into subsets. The choices for $p, q, r$ and the four possible placements of "l" in the Wythoff cases,

$$
p|q r, \quad p q| r, \quad p q r|, \quad| p q r
$$

suffice to describe the constructions for all uniform polyhedra except for the one nonWythoffian case. The relative placements of "|" denote the four ways to select a point $C$ in spherical triangle ( $p q r$ ) so that it traces the vertices of regular polygonal faces all having the same edge-length:

$$
\begin{array}{ll}
p \mid q r & C \text { is the vertex } P \text { of }(p q r) . \\
p q \mid r & C \text { lies on the arc } P Q \text { of }(p q r) \text { and on the bisector of the opposite angle } R . \\
p q r \mid & C \text { is the incenter of }(p q r) \text { which is equidistant from the triangle's three } \\
\text { sides. } & \begin{array}{l}
C \text { is a special point in }(p q r) \text { whose images under taking an even number } \\
\text { of reflections trace a snub polyhedron. The location of } C \text { depends on the } \\
\text { solution of the quartic equation (4) in Section } 6 .
\end{array}
\end{array}
$$

It should be stressed that Wythoff constructions can be applied to any point $C$ of a Schwarz triangle ( $p q r$ ), leading to isogonal (vertex-transitive) polyhedra. However, only with the special choices of $C$ listed above will the resulting faces be regular polygons, and the polyhedra uniform. Furthermore, only if $p, q, r$ are all integers will the resulting uniform polyhedra be convex.

Varying the order of the numbers within a subset of $p, q, r$ does not affect the kind of uniform polyhedron. Excluding such redundancies, the other permutations of Wythoff symbols (using "|" and the set of nine rational numbers) do not always produce new or valid polyhedra as some are degenerate forms. Consider, for example, that the regular octahedron 4 | 23 can also be interpreted as 2 | 33 or | 223. Such equivalencies are made apparent in the next section where we derive the cycle of faces that surround each vertex.

The final tally of uniform polyhedra has been proven to be only 77 [33]-[35]. They are listed in Appendix B for quick reference and the complete set is figured in [7], [14], [18], [38], and [39]. One can find instructive images of all the uniform polyhedra and their duals on the Internet, some of which are virtual reality models that the user can spin [3].

\section{The Vertex Figure and Vertex Cycle of a Uniform Polyhedron}

It is desirable to regard the vertices of the "vertex figure" as lying at unit distance from one vertex of the polyhedron along all edges incident to this vertex. In this respect, every uniform polyhedron has a characteristic planar vertex figure which is a cyclic polygon having a signed side $v_{n}=2 \cos (\pi / n)$ corresponding to each regular $n$-gonal face incident to a vertex of the polyhedron. The circumradius of the vertex figure is denoted by $\rho$.

A uniform polyhedron is frequently described by its vertex cycle symbol $n_{1} \cdot n_{2} \cdots \cdots \cdot n_{k}$ where $\left\{n_{1}\right\},\left\{n_{2}\right\}, \ldots,\left\{n_{k}\right\}$ are the $k$ faces that surround each vertex. We use $\{n\}$ to denote a regular $n$-gonal face for reduced rational $n>1$. Appendix B lists the 77 Wythoff symbols together with the corresponding vertex cycle symbols which tend to be more 
intuitive than the former. The relation between the two kinds of symbols is established by the following observations:

If $n>2$, then $\{n\}$ is an "ordinary" face and the corresponding positive side, $v_{n} \geq 0$, of the vertex figure subtends a positive (counterclockwise) central angle.

If $n=2$, then the side of the vertex figure corresponding to the digon face reduces to a point. Hence $\{2\}$ is eliminated from the cycle of faces whenever it appears in the conversion of Wythoff symbol to vertex cycle symbol.

If $1<n<2$, then $\{n\}$ is a "retrograde" face which corresponds to a negative side, $v_{n}<0$, and negative central angle.

Some published vertex cycles give the ordinary notations $n>2$ for faces that are actually retrograde. It is important to recognize and preserve the two basic kinds of $\{n\}$ that compose the vertex cycle as certain calculations depend on this distinction. If the retrograde form of ordinary $\{n\}$ is denoted $\left\{n^{\prime}\right\}$, then $n^{\prime}=n /(n-1)$ and so we have the complementary relation $1 / n+1 / n^{\prime}=1$. For example, $\left\{\frac{5}{3}\right\}$ is the retrograde form of $\left\{\frac{5}{2}\right\}$. It is further observed that the ordinary or retrograde face $\{n\}$ has num $(n)$ edges and has a polygonal density that is the denominator of the ordinary form of $n$. Here and throughout, $\operatorname{num}(x)$ denotes the largest numerator in a set $x=\left\{n_{1}, n_{2}, \ldots\right\}$ of reduced rational numbers $n_{j}$.

Figure 1 illustrates retrograde triangle faces $\left\{\frac{3}{2}\right\}$ of the uniform polyhedron with Wythoff symbol $\frac{3}{2} 4 \mid 4$ and vertex cycle symbol $\frac{3}{2} \cdot 8 \cdot 4 \cdot 8$. Figure 2 is the associated vertex figure which is inscribed in the circle of radius $\rho$ and which shows retrograde winding of the side that corresponds to $\left\{\frac{3}{2}\right\}$. Figure 2 could be interpreted as having vertex cycle $3 \cdot 8 \cdot \frac{4}{3} \cdot 8$ with retrograde square faces. However, the latter vertex cycle symbol would need to associate with Wythoff symbol $3 \frac{4}{3} \mid 4$ which essentially leads

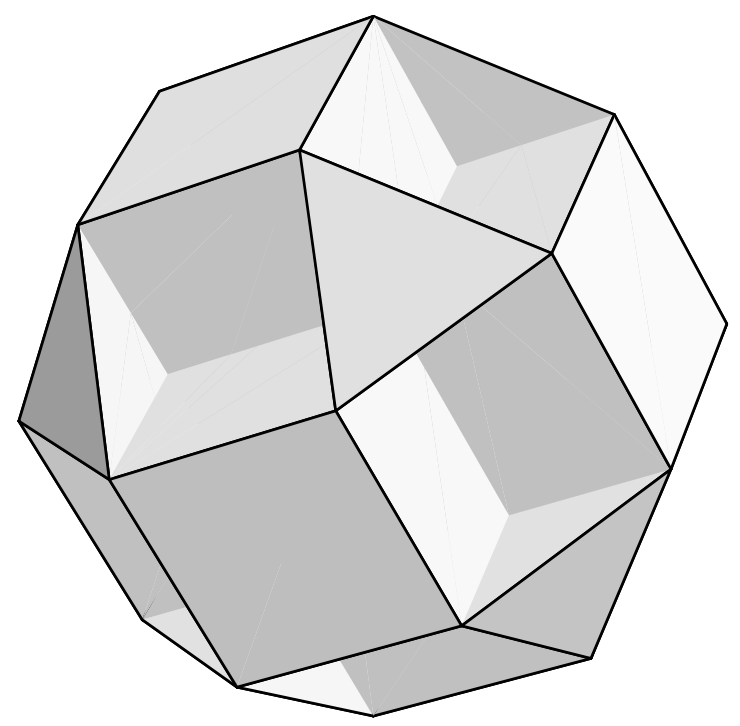

Fig. 1. Uniform polyhedron $\frac{3}{2} 4 \mid 4$ with vertex cycle $\frac{3}{2} \cdot 8 \cdot 4 \cdot 8$. 


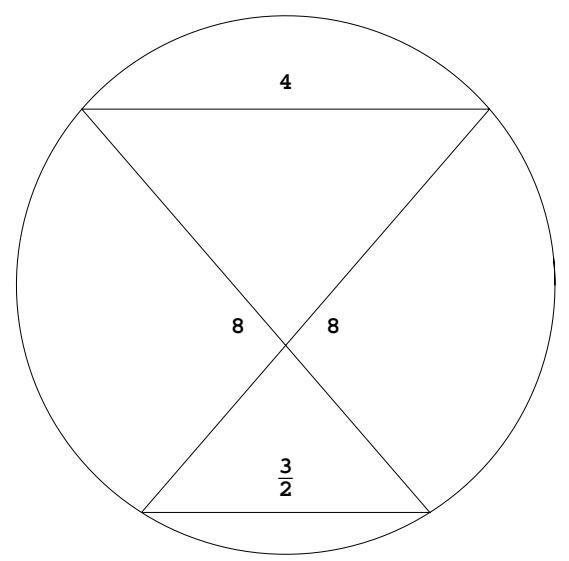

Fig. 2. Vertex figure of $\frac{3}{2} 4 \mid 4$.

to the same polyhedron as $\frac{3}{2} 4 \mid 4$. For such equivalencies Coxeter et al. [7] discard the Wythoff symbol derived from the larger colunar Schwarz triangle (see Section 5). The foregoing associations are made clear as we now summarize the conversion of Wythoff symbol to vertex cycle symbol.

$p \mid q r$ yields vertex cycle $(q \cdot r)^{p}$ which expands to $q \cdot r \cdot q \cdot r \cdot, \ldots, q \cdot r$. By expressing $p$ as the reduced fraction $n_{p} / d_{p}$, we observe that portion $q \cdot r$ is repeated $n_{p}$ times in the complete vertex cycle that winds $d_{p}$ times around the polyhedral vertex.

$p q \mid r$ yields simply vertex cycle $p \cdot 2 r \cdot q \cdot 2 r$.

If all denominators (including 1) of $p, q, r$ are odd, then $p q r \mid$ yields the vertex cycle $2 p \cdot 2 q \cdot 2 r$ except when an angle of the (triangular) vertex figure is obtuse. If this is the angle opposite $v_{2 r}$ so that $\left(v_{2 p}\right)^{2}+\left(v_{2 q}\right)^{2}<\left(v_{2 r}\right)^{2}$, then the cycle is $(2 p)^{\prime} \cdot(2 q)^{\prime} \cdot 2 r$, where $n^{\prime}=n /(n-1)$.

If just one of $p, q, r$ in $p q r \mid$ has an even denominator, let this be $r$. It follows that the incenter $C$ of spherical triangle $P Q R$ lies on the bisector at $R$ which also corresponds to a reflection plane in the symmetry group. In the course of tracing $\{2 p\}$ and $\{2 q\}$ about centers $P$ and $Q$, respectively, the images of $C$ reflected across $R P$ and $R Q$ trace a $\{2 r\}$ twice about center $R$. This leads to the curious vertex cycle $2 r \cdot 2 p \cdot 2 q \cdot 2 r \cdot 2 q \cdot 2 p$ consisting of two $\{2 p\}$ 's, two $\{2 q\}$ 's, and a $\{2 r\}$ traversed twice. By discarding the double $\{2 r\}$ we are left with an acceptable one-sided polyhedron whose vertex figure is a crossed quadrilateral ("butterfly"). In traversing the crossed quadrilateral we notice a reversal in direction and so the vertex cycle is $2 p \cdot 2 q \cdot(2 p)^{\prime} \cdot(2 q)^{\prime}$. For these cases of $p q r$ । Coxeter et al. [7] use the hybrid notation

$$
p q{ }_{s}^{r}
$$

where $v_{2 r}$ and $v_{2 s}$ are the distances spanned by the two vacant bases of the crossed quadrilateral vertex figure. While not a true Wythoff symbol, the hybrid notation is useful as the same polyhedron is derived from $p q s \mid$ where $s$ too has an even denominator. The quantities are related by Ptolemy's theorem: $\operatorname{sgn}(q-p) v_{2 r} v_{2 s}=\left(v_{2 q}\right)^{2}-\left(v_{2 p}\right)^{2}$. Here and throughout, $\operatorname{sgn}(x)=1$ for $x \geq 0$ while $\operatorname{sgn}(x)=-1$ for $x<0$. For convenience, 
the hybrid notations are included in Appendix B instead of the separate Wythoff symbols $p q r \mid$ and $p q s \mid$.

Snub notation $\mid p q r$ yields vertex cycle $p \cdot t \cdot q \cdot t \cdot r \cdot t$ where intercalating $t$ is $t=3$ or $\frac{3}{2}$. A useful algorithm for $t$ is $t=6 /(3-\operatorname{sgn}(\rho-\lambda / \sqrt{3}))$. Here and throughout, $\lambda=0$ or 1 depending on whether the Wythoff symbol signifies dihedral symmetry or not dihedral symmetry, respectively. Dihedral symmetry exists when 2 occurs twice among the Wythoff elements, that is, uniform prisms $p 2 \mid 2$ and antiprisms $\mid p 22$. We observe that when $\lambda=1$, an occurrence of element $\frac{3}{2}$ in $\mid p q r$ forces the intercalating triangles in the vertex cycle to be retrograde, $t=\frac{3}{2}$, in order to preserve the consistency of signed measurements used in later formulas. The effect of circumradius $\rho$ of the vertex figure is further discussed in Section 6.

The pseudo-Wythoff symbol $\mid p q r s$ identifies the sole non-Wythoffian uniform polyhedron $\mid 3 \frac{5}{2} \frac{3}{2} \frac{5}{3}$ which yields vertex cycle $3 \cdot 4 \cdot \frac{5}{2} \cdot 4 \cdot \frac{3}{2} \cdot 4 \cdot \frac{5}{3} \cdot 4$.

A useful exercise in reverse is to determine the Wythoff symbol directly from a given cycle of the various $\{n\}$ surrounding a vertex.

\section{Enumerative Quantities}

Order $g$ of the full symmetry group for Schwarz triangle $\left(\begin{array}{l}p q r \\ \mathrm{r}\end{array}\right)$ is given by $g=$ $4 d /(1 / p+1 / q+1 / r-1)$ where density $d$ for the higher polyhedral symmetries is discussed in Section 2 . This expression also applies to dihedral symmetry where we find $d$ to be the denominator of $p$ in ( $\left.\begin{array}{l}p 2 \\ 2\end{array}\right)$. For programming purposes a useful algorithm that does not require $d$ is $g=(4)\left(6^{\lambda}\right)(\eta+\lambda(\eta-2)(\eta-5))$ where $\eta=\operatorname{num}(p, q, r)$ and $\lambda=0$ or 1 as defined in the previous section. A figure that possesses at least one plane of symmetry is said to be "reflexible." Thus, a reflexible uniform polyhedron has either dihedral symmetry $(\eta>2, g=4 \eta)$, tetrahedral symmetry $(\eta=3, g=24)$, octahedral symmetry $(\eta=4, g=48)$, or icosahedral symmetry $(\eta=5, g=120)$. Non-reflexible snubs cases $\mid p q r$ and $\mid p q r s$ have rotational symmetries of order $g / 2$.

Various combinatorial and metrical quantities associated with uniform polyhedra can be calculated directly from their Wythoff symbols. We begin by enumerating the vertices, edges, and faces of a uniform polyhedron. Denoting $N_{0}=$ number of vertices, $N_{1}=$ number of edges, and $N_{2}=$ total number of faces which consist of $i$ faces of form $\{m\}$, $j$ faces of form $\{n\}, \ldots$ where $i+j+\cdots=N_{2}$, we then have:

\begin{tabular}{cl}
\hline Wythoff symbol & \multicolumn{1}{c}{$N_{o}$} \\
\hline$p \mid q r$ & $\frac{g}{2 \text { num }(p)}$ \\
$p q \mid r$ & $\frac{g}{4}$ if $\frac{1}{p}+\frac{1}{q}=1$ and $r<\frac{g}{8} ;$ otherwise $\frac{g}{2}$ \\
$p q r \mid$ & $g$ \\
$p q r \mid$ & $\frac{g}{2}$ \\
$s \mid$ & $\frac{g}{2}$ \\
$\mid p q r$ & $\frac{g}{2}$ \\
$\mid p q r s$ &
\end{tabular}




$$
N_{1}=\frac{N_{0} N_{01}}{2}
$$

where $N_{01}$ is the number of edges at each vertex, which is the number of elements in the vertex cycle denoted by $k$ in Section 3 [7].

$$
{ }_{n} N_{2}=\frac{N_{0} N_{02}}{\operatorname{num}(n)}
$$

is the number of face kind $\{n\}$ where $N_{02}$ is the number of occurrences of $\{n\}$ in the vertex cycle [7].

$$
N_{2}=\sum{ }_{n} N_{2}
$$

represents the sum of ${ }_{n} N_{2}$ over the (non-repeated) kinds of $n$ that occur in the vertex cycle.

\section{A Vertex of a Non-Snub Uniform Polyhedron}

In this section and the next we present expressions for coordinates of vertices of the various uniform polyhedra. The main part of this is the determination of one vertex in relation to the symmetry group of the polyhedron. We begin here with the non-snub uniform polyhedra.

The location of the initial vertex $(x, y, z)$ depends on the specific Cartesian orientation of the spherical Schwarz triangle ( $p q r)$ that contains the vertex. The specific orientation of ( $p q r$ ), geometrically denoted as $P Q R$, is such that $R$ lies on the $Z$-half-axis: $Z>0$; $P$ lies in the $X Z$-half-plane: $X>0 ; Q$ lies in the $X Y Z$-half-space: $Y>0$.

The expression

$$
N=\sqrt{-\cos S \cos (S-A) \cos (S-B) \cos (S-C)}
$$

is encountered in advanced treatments [36] of spherical triangle $A B C$ where half the sum of its angles is $S=(A+B+C) / 2$. We instead use a more convenient expression $H=2 N$ in discussing the uniform polyhedra. Accordingly, quantity $H^{2}$ is expressed in terms of the Wythoff elements by any of these symmetrical forms:

$$
\begin{aligned}
H^{2}= & \left|\begin{array}{ccc}
1 & -\cos \frac{\pi}{q} & -\cos \frac{\pi}{r} \\
-\cos \frac{\pi}{q} & 1 & -\cos \frac{\pi}{p} \\
-\cos \frac{\pi}{r} & -\cos \frac{\pi}{p} & 1
\end{array}\right| \\
= & 1-\cos ^{2} \frac{\pi}{p}-\cos ^{2} \frac{\pi}{q}-\cos ^{2} \frac{\pi}{r}-2 \cos \frac{\pi}{p} \cos \frac{\pi}{q} \cos \frac{\pi}{r} \\
= & -4 \cos (S) \cos \left(S-\frac{\pi}{p}\right) \cos \left(S-\frac{\pi}{q}\right) \cos \left(S-\frac{\pi}{r}\right) \\
& \text { where } S=\frac{\pi}{2}\left(\frac{1}{p}+\frac{1}{q}+\frac{1}{r}\right) .
\end{aligned}
$$


An interesting known relation for example is

$$
N^{2}=\frac{H^{2}}{4}=\cot \left(R_{0}\right) \cot \left(R_{1}\right) \cot \left(R_{2}\right) \cot \left(R_{3}\right)
$$

where $R_{0}$ is the angular radius of the small circle circumscribed about $P Q R$ and $R_{1}, R_{2}, R_{3}$ are the angular radii of the circumcircles for each of the three colunar triangles of $P Q R$. The three colunar triangles of $P Q R$ are Schwarz triangles $P^{\prime} Q R, P Q^{\prime} R, P Q R^{\prime}$, where $P^{\prime}, Q^{\prime}, R^{\prime}$ are antipodes of $P, Q, R$, respectively. More relationships involving $H$ appear in Section 9.

We begin by scaling the oriented spherical triangle $P Q R$ so that $P, Q, R$ are each unit distance from the origin. Using standard methods of spherical trigonometry and analytic geometry, we determine the Cartesian coordinates of $P, Q, R$ to be

$$
\begin{aligned}
& P: \quad\left(\frac{H}{\sin (\pi / r) \sin (\pi / p)}, 0, \frac{\cos (\pi / q)+\cos (\pi / r) \cos (\pi / p)}{\sin (\pi / r) \sin (\pi / p)}\right), \\
& Q: \quad\left(\frac{H}{\sin (\pi / q) \tan (\pi / r)}, \frac{H}{\sin (\pi / q)}, \frac{\cos (\pi / p)+\cos (\pi / q) \cos (\pi / r)}{\sin (\pi / q) \sin (\pi / r)}\right), \\
& R: \quad(0,0,1) .
\end{aligned}
$$

From $P, Q, R$ we can next determine the normal equations for the three planes (Schwarz planes) determined by sides $R P, R Q, P Q$ of $P Q R$ as follows:

Plane RP: $\quad y=0$.

Plane $R Q: \quad\left(\sin \frac{\pi}{r}\right) x-\left(\cos \frac{\pi}{r}\right) y=0$.

Plane $P Q: \quad\left(\frac{-\cos (\pi / q)-\cos (\pi / p) \cos (\pi / r)}{\sin (\pi / r)}\right) x-\left(\cos \frac{\pi}{p}\right) y+\left(\frac{H}{\sin (\pi / r)}\right) z=0$.

Depending on the choice of Wythoff case, the vertex $(x, y, z)$ will either be distance 0 or 1 from each of the Schwarz planes assuming edge-length 2 . Thus $(x, y, z)$ is the simultaneous solution of three distances from the three Schwarz planes. The vertex coordinates $(x, y, z)$ in terms of the specific order $p, q, r$ and Wythoff case type $\sigma_{i}$ are generalized as follows:

$$
\begin{aligned}
& x=\frac{1+\sigma_{1} \cos (\pi / r)}{\sin (\pi / r)} ; \quad y=\sigma_{1} \\
& z=\left(\left(\cos \frac{\pi}{q}+\cos \frac{\pi}{p} \cos \frac{\pi}{r}\right) x+\left(\sigma_{2}+\cos \frac{\pi}{p}\right)\left(\sin \frac{\pi}{r}\right) y\right) / H,
\end{aligned}
$$

where $\sigma_{1}=0$ for the first Wythoff case $p \mid q r$, otherwise $\sigma_{1}=1$; and $\sigma_{2}=0$ for the second Wythoff case $p q \mid r$, otherwise $\sigma_{2}=1$. The remaining vertices may be traced by successive reflections across the Schwarz planes of $P Q R$. More specifically, the reflection of the point $\left(x_{0}, y_{0}, z_{0}\right)$ across a plane with normal equation form

$$
u_{1} x+u_{2} y+u_{3} z=0: u_{1}^{2}+u_{2}^{2}+u_{3}^{2}=1
$$

is the image point $\left(x_{0}-u_{1} f, y_{0}-u_{2} f, z_{0}-u_{3} f\right)$ where $f=2\left(u_{1} x_{0}+u_{2} y_{0}+u_{3} z_{0}\right)$. In the literature this image is usually expressed as the product of a vector and a $3 \times 3$ matrix 
$M$ of reflection. Accordingly, the image of the point $\left(x_{0}, y_{0}, z_{0}\right)$ is the point $\left[x_{0} y_{0} z_{0}\right] M$ where

$$
M=\left[\begin{array}{ccc}
1-2 u_{1}^{2} & -2 u_{1} u_{2} & -2 u_{1} u_{3} \\
-2 u_{2} u_{1} & 1-2 u_{2}^{2} & -2 u_{2} u_{3} \\
-2 u_{3} u_{1} & -2 u_{3} u_{2} & 1-2 u_{3}^{2}
\end{array}\right] .
$$

An interesting problem for each uniform polyhedron is to map a Hamiltonian circuit of non-repeated vertices among the $N_{0}$ vertices using only the three Schwarz planes of $P Q R$ as reflection planes. Starting with vertex $\left(x_{0}, y_{0}, \mathrm{z}_{0}\right)$, such Hamiltonian circuits are generated by certain orders of successive kaleidoscopic reflections with the modification that vertices of snub polyhedra are produced by the even reflections (see Section 2). Difficulty arises from the fact that the necessary order of reflections across the three Schwarz planes cannot be regular repetitions.

\section{A Vertex of the Snub Uniform Polyhedron $\mid p q r$}

The rules for orienting $P Q R$ of $\mid p q r$ are outlined in Section 5. To determine the coordinates of a snub vertex in $P Q R$ we begin with the signed sides $v_{n}$ of the vertex figure as defined in Section 3:

$$
a=v_{p}=2 \cos \frac{\pi}{p} ; \quad b=v_{q}=2 \cos \frac{\pi}{q} ; \quad c=v_{r}=2 \cos \frac{\pi}{r} .
$$

Then from $H^{2}$ as specified in (1) we make use of $4 H^{2}=4-a^{2}-b^{2}-c^{2}-a b c$.

The location of the initial snub point $C$ in $P Q R$ requires that its reflections in the side planes of $P Q R$ produce the vertices of the appropriate equilateral triangle $C^{\prime} C^{\prime \prime} C^{\prime \prime \prime}$ which corresponds to the snub $\{3\}$ of $\mid p q r$. Point $C$ is then a vertex of another $\mid p q r$ which is enantiomorphic to the snub polyhedron with face $C^{\prime} C^{\prime \prime} C^{\prime \prime \prime}$. Coxeter et al. [7] obtain a natural coordinate system by letting $\boldsymbol{x}, \boldsymbol{y}, \boldsymbol{z}$ denote the distances of $C$ from the three planes and show that the conditions for snub $\{3\}$ require $x^{2}-a y z=y^{2}-b z x=z^{2}-c x y$. Eliminating $z=\left(\boldsymbol{x}^{2}-\boldsymbol{y}^{2}\right) /(a \boldsymbol{y}-b \boldsymbol{x})$, Coxeter et al. obtain a quartic equation in variables $\boldsymbol{x}$ and $\boldsymbol{y}$ which we modify here by setting $\boldsymbol{y}=1$ and changing $\boldsymbol{x}$ to our $w$ :

$$
\left(1-b^{2}\right) w^{4}+b(a-b c) w^{3}+2(a b c-1) w^{2}+a(b-a c) w+1-a^{2}=0 .
$$

The Cartesian coordinates $\left(x_{w}, y_{w}, z_{w}\right)$ of vertex $C$ are derived from the simultaneous solution of the three distances $w, 1,\left(w^{2}-1\right) /(a-b w)$ from $C$ to the sides of $P Q R$, whose equations are specified in (2). The case of two positive quartic roots is discussed at the end of this section.

A convenient set of vertex coordinates $\left(x_{w}, y_{w}, z_{w}\right)$ for $\mid p q r$ scaled to edge-length

$$
e_{w}=4 \sin \frac{\pi}{r} \sqrt{w^{2}+c w+1}=2 \sin \frac{\pi}{r} \sqrt{x_{w}^{2}+y_{w}^{2}}
$$

is

$$
\begin{gathered}
x_{w}=2 w+c ; \quad y_{w}=2 \sin \frac{\pi}{r}=\sqrt{4-c^{2}} \\
z_{w}=\frac{\left(a^{2}-b^{2}\right)\left(w^{2}+c w+1\right)+4 H^{2}\left(w^{2}-1\right)}{2 H(a-b w)} .
\end{gathered}
$$


Thus, vertex $\left(k x_{w}, k y_{w}, k z_{w}\right): k=2 / e_{w}$ corresponds to the snub polyhedron of edgelength 2 .

As an alternate approach to determining $k$, consider angle $\phi$ at the polyhedron's center which is subtended by a half-edge and circumsphere radius ${ }_{0} R$ as defined in Section 8. If a vertex $\left(x_{w}, y_{w}, z_{w}\right)$ in $P Q R$ is oriented as described, then specifically for snub uniform polyhedra we have the relation

$$
\cos \phi=\sqrt{\cos ^{2} \frac{\pi}{r}+\sin ^{2} \frac{\pi}{r}\left(\frac{z_{w}^{2}}{x_{w}^{2}+y_{w}^{2}+z_{w}^{2}}\right)}
$$

and so $\rho=\cos \phi$ when the edge-length is 2 . Then from (6) we have

$$
{ }_{0} R=\frac{1}{\sqrt{1-\rho^{2}}}=\frac{1}{\sin (\pi / r) \sqrt{1-z_{w}^{2} /\left(x_{w}^{2}+y_{w}^{2}+z_{w}^{2}\right)}}
$$

and so the desired vertex is $\left(k x_{w}, k y_{w}, k z_{w}\right): k={ }_{0} R / \sqrt{x_{w}^{2}+y_{w}^{2}+z_{w}^{2}}$ which applies to snub polyhedra of edge-length 2 .

A snub polyhedron is reflexible if two of the Wythoff elements $p, q, r$ are identical. Unfortunately quartic equation (4) fails to produce a viable root when $p=q$ for the first two ordered elements. For example, if the Wythoff symbol $\mid p q r$ is such that $p=q=3$ or $p=q=\frac{3}{2}$, then quartic equation (4) reduces to the insoluble case $w^{2}-2 w+1=0$, that is, $w=1$ regardless of $r$. Accordingly, the reflexible snub $\mid \frac{3}{2} \frac{3}{2} \frac{5}{2}$ must be taken as $\mid \frac{5}{2} \frac{3}{2} \frac{3}{2}$ which is obtained by permuting the elements of the Wythoff symbol. Here different permutations of $p, q, r$ produce the same polyhedron but with different Cartesian orientation and with different $e_{w}$. Thus, one simple way to resolve a case of two simultaneous positive quartic roots is to permute the cycle of elements $p, q, r$ and then reapply the quartic equation until one positive root occurs. However, a more direct method is to select the one positive root which gives $\rho$ from $\left(x_{w}, y_{w}, z_{w}\right)$ so that the algebraic sum of the central angles subtended by the sides of the corresponding vertex figure is a multiple of $2 \pi$. This condition is satisfied by

$\sin \left(\operatorname{sgn}\left(\rho-\frac{\lambda}{\sqrt{3}}\right) 3 \arcsin \left(\frac{1}{2 \rho}\right)+\arcsin \left(\frac{a}{2 \rho}\right)+\arcsin \left(\frac{b}{2 \rho}\right)+\arcsin \left(\frac{c}{2 \rho}\right)\right)=0$.

The hypothetical case $\mid 222$ produces an equilateral triangle vertex figure of $\rho=1 / \sqrt{3}$ which we interpret to be a pivotal state between vertex figures that encircle the center once or twice. Thus, we observe that for $\mid p q r$ when $\lambda=1$, condition $\rho \geq 1 / \sqrt{3}$ relates to snub triangles $\{3\}$ and central angle sum $2 \pi$ while condition $\rho<1 / \sqrt{3}$ relates to snub triangles $\left\{\frac{3}{2}\right\}$ and central angle sum $-4 \pi$.

\section{A Vertex of the Sole Uniform Polyhedron $\mid p q r s$}

Non-Wythoffian case | $3 \frac{5}{2} \frac{3}{2} \frac{5}{3}$ is closely related to snub case | $3 \frac{5}{2} \frac{5}{3}$ in that both polyhedra share the same vertices. Thus, we obtain a non-Wythoffian vertex by applying the previous formulas to the related snub case. 


\section{Metrical Quantities of the Orthoschemes}

The orthoscheme corresponding to each $\{n\}$ of the uniform polyhedron is a "quadrirectangular" tetrahedron $\mathrm{O}_{0} \mathrm{O}_{1} \mathrm{O}_{2} \mathrm{O}_{3}$ which has four right triangle faces, $\mathrm{O}_{0} \mathrm{O}_{1} \mathrm{O}_{2}, \mathrm{O}_{0} \mathrm{O}_{1} \mathrm{O}_{3}$, $\mathrm{O}_{0} \mathrm{O}_{2} \mathrm{O}_{3}, \mathrm{O}_{1} \mathrm{O}_{2} \mathrm{O}_{3}$, and three mutually perpendicular edges, $\mathrm{O}_{0} \mathrm{O}_{1}, \mathrm{O}_{1} \mathrm{O}_{2}, \mathrm{O}_{2} \mathrm{O}_{3}$, where $\mathrm{O}_{0}$ is a vertex of the polyhedron, $O_{1}$ is the midpoint of an edge, $O_{2}$ is the center of $\{n\}$, and $\mathrm{O}_{3}$ is the center of the polyhedron. Angle $\mathrm{O}_{0} \mathrm{O}_{2} \mathrm{O}_{1}$ is simply $\pi / n$, the angle subtended by a half-edge at the center of $\{n\}$. Subsequent expressions are simplified if we set half-edge $O_{0} O_{1}$ to 1 and use the following notations:

$$
\begin{array}{ll}
{ }_{0} r_{n}=O_{0} O_{2}, & \text { the circumradius of }\{n\} . \\
{ }_{1} r_{n}=O_{1} O_{2}, & \begin{array}{l}
\text { the perpendicular distance (apothem) to the side of }\{n\} \text { from the } \\
\text { polygonal center. } \\
{ }_{0} R=O_{0} O_{3},
\end{array} \\
{ }_{1} R=O_{1} O_{3}, & \text { the circumsphere radius. } \\
{ }_{2} R_{n}=O_{2} O_{3} & \text { the midsphere radius. } \\
\phi=O_{0} O_{3} O_{1}, & \text { the angle at } O_{3} \text { subtended by a half-edge. } \\
\chi_{n}=O_{0} O_{3} O_{2}, & \text { the angle at } O_{3} \text { subtended by }{ }_{0} r_{n} . \\
\psi_{n}=O_{1} O_{3} O_{2}, & \text { the angle at } O_{3} \text { subtended by }{ }_{1} r_{n} .
\end{array}
$$

$\delta_{m, n}=$ the dihedral angle $0<\delta_{m, n}<\pi$ at the edge shared by $\{m\}$ and $\{n\}$ where the distinction between an ordinary and a retrograde face is important. $\delta_{m, n}$ is that dihedral angle which encloses $\mathrm{O}_{3}$ even if $\mathrm{O}_{3}$ is contained by one of the faces. If one face is retrograde but not the other, then the dihedral angle $\pi-\delta_{m, n}$ encloses a "locally interior" portion of the polyhedron.

Beginning with this section, quantities are expressed in terms of $\pi / n$ and $\phi$. More specifically, we favor using the trigonometric constants $\cot (\pi / n)$ and $\cot \phi$ because they frequently provide the most concise expressions. This consistency will expedite the task of computer programming.

For any vertex $(x, y, z)$ of a uniform polyhedron of edge-length 2 , we derive these familiar right triangle relationships of the orthoscheme:

$$
\begin{gathered}
{ }_{0} R=\sqrt{x^{2}+y^{2}+z^{2}}=\sqrt{\cot ^{2} \phi+1}=\csc \phi=\frac{1}{\sqrt{1-\rho^{2}}} ; \\
{ }_{1} R=\cot \phi=\sqrt{{ }_{0} R^{2}-1} ; \quad \rho=\cos \phi=\frac{{ }_{1} R}{{ }_{0} R}=\sqrt{1-\frac{1}{{ }_{0} R^{2}}}
\end{gathered}
$$

A retrograde element $n^{\prime}$ of the vertex cycle must first be changed to its ordinary complement form $n$ for this set of calculations:

$$
\begin{gathered}
{ }_{1} r_{n}=\cot \frac{\pi}{n} ; \quad{ }_{0} r_{n}=\csc \frac{\pi}{n}=\sqrt{\cot ^{2} \frac{\pi}{n}+1} ; \quad{ }_{2} R_{n}=\sqrt{\cot ^{2} \phi-\cot ^{2} \frac{\pi}{n}} \\
\sin \chi_{n}=\sin \phi \csc \frac{\pi}{n}=\sqrt{\frac{\cot ^{2}(\pi / n)+1}{\cot ^{2} \phi+1}} ; \quad \sin \psi_{n}=\frac{\cot (\pi / n)}{\cot \phi}
\end{gathered}
$$


A retrograde element is not changed to its ordinary form for dihedral angle calculations [22]:

$$
\cos \delta_{m, n}=\frac{\cot (\pi / m) \cot (\pi / n)-\sqrt{\left(\cot ^{2} \phi-\cot ^{2}(\pi / m)\right)\left(\cot ^{2} \phi-\cot ^{2}(\pi / n)\right)}}{\cot ^{2} \phi} .
$$

Note that

$$
\beta_{m, n}=\left|\arccos \left(\frac{\cot (\pi / m)}{\cot \phi}\right)+\arccos \left(\frac{\cot (\pi / n)}{\cot \phi}\right)\right|
$$

is an expression which reveals the sum of the two component dihedral angles that occur between a common plane through $O_{3}$ and the corresponding $n$-gon facial plane. Thus $\delta_{m, n}$ is the lesser of $\beta_{m, n}$ or $2 \pi-\beta_{m, n}$.

\section{Expressions for $\phi$ in Terms of the Wythoff Elements}

Using the non-snub initial vertex $(x, y, z)$ specified in (3) and the relation $\cot ^{2} \phi=$ $x^{2}+y^{2}+z^{2}-1$ derived from (6), we express $\cot \phi$ in terms of the three elements $p, q, r$ in the Wythoff symbol. Quantity $H$ from (1) in Section 5 appears as a factor in the compact relations that follow. Deeper significance of this quantity is seen in the relation $H=\sin (\pi / h)$ where the definition of "Coxeter number" $h$ in [5] is extended to the general Schwarz triangle $(p q r)$ such that $\cos ^{2}(\pi / h)=\cos ^{2}(\pi / p)+\cos ^{2}(\pi / q)+$ $\cos ^{2}(\pi / r)+2 \cos (\pi / p) \cos (\pi / q) \cos (\pi / r)$ in [18].

Rational values for $h$ relate to the symmetry group of the Schwarz triangle as follows: dihedral (2 $2 p$ ): $h=\max \left(p, p^{\prime}\right)$; tetrahedral: $h=4$; octahedral: $h=6$; icosahedral: $h=6,10$, or $\frac{10}{3}$. The corresponding values for $H$ are

tetrahedral: $\quad H=\frac{1}{\sqrt{2}} ; \quad$ octahedral: $\quad H=\frac{1}{2} ; \quad$ icosahedral: $\quad H=\frac{1}{2}, \frac{1}{2 \tau}$, or $\frac{\tau}{2}$,

where $\tau=(1+\sqrt{5}) / 2$ is the golden ratio.

We first express $\cot \phi$ for all non-snub uniform polyhedra as

$$
\begin{aligned}
\cot \phi=\csc \frac{\pi}{r} & {\left[\left(\sigma_{1}+\cos \frac{\pi}{r}\right)^{2}+\left(1 / H^{2}\right)\left(\cos \frac{\pi}{p}\left(\sigma_{1}+\cos \frac{\pi}{r}\right)\right.\right.} \\
& \left.\left.+\cos \frac{\pi}{q}\left(1+\sigma_{1} \cos \frac{\pi}{r}\right)+\sigma_{1} \sigma_{2} \sin ^{2} \frac{\pi}{r}\right)^{2}\right]^{1 / 2},
\end{aligned}
$$

where $\sigma_{1}=0$ for the first Wythoff case $p \mid q r$, otherwise $\sigma_{1}=1$; and $\sigma_{2}=0$ for the second Wythoff case $p q \mid r$, otherwise $\sigma_{2}=1$.

The right side of (7) can be simplified for specific Wythoff cases as follows:

For case $p \mid q r$,

$$
\cot \phi=\sqrt{\frac{1}{H^{2}} \sin ^{2} \frac{\pi}{p}-1}
$$


from which we derive the compact relation

$$
\sin \phi=\csc \frac{\pi}{p} \sin \frac{\pi}{h} .
$$

For case $p q \mid r$,

$$
\cot \phi=\frac{1}{H} \sqrt{\left(1+\cos \frac{\pi}{r}\right)\left(1+\cos \frac{\pi}{r}+2 \cos \frac{\pi}{p} \cos \frac{\pi}{q}\right)}
$$

from which we derive

$$
\sin \phi=\frac{\sin (\pi / h)}{\sqrt{(2 \cos (\pi / 2 r))^{2}-(\cos (\pi / p)-\cos (\pi / q))^{2}}} .
$$

For cases $p q r \mid$ and $p q_{s}^{r} \mid$,

$$
\cot \phi=\frac{4}{H} \cos \frac{\pi}{2 p} \cos \frac{\pi}{2 q} \cos \frac{\pi}{2 r}=4 \cos \frac{\pi}{2 p} \cos \frac{\pi}{2 q} \cos \frac{\pi}{2 r} \csc \frac{\pi}{h},
$$

where $s$ may be substituted for $r$.

For the snub case | $p q r$ we make use of the vertex figure sides defined in Section 3 by assigning

$$
\begin{array}{ll}
a=v_{p}=2 \cos \frac{\pi}{p}, & b=v_{q}=2 \cos \frac{\pi}{q}, \quad c=v_{r}=2 \cos \frac{\pi}{r}, \\
s=a^{2}+b^{2}+c^{2}, & t=a b c, \quad \text { and } \quad u=a^{2} b^{2}+b^{2} c^{2}+c^{2} a^{2} .
\end{array}
$$

Then $\cot \phi=(2-Y)^{-1 / 2}$ where $Y$ is a real root of

$$
(1-t) Y^{4}+(3 t-u) Y^{3}+(3 u-2 s-s t) Y^{2}+t(3 s-t-8) Y+s^{2}+3 t^{2}-4 u=0 .
$$

A similar fourth-degree "snub equation" has been described by Coxeter et al. [7, equation 10.3] in the variable $X=1-\tan ^{2} \phi$. It is simplified to (8) in the variable $Y=2-\tan ^{2} \phi$ by making the substitution $X=Y-1$. From relations (6) in Section 8, other important quantities can now be expressed in terms of $Y$, for example $\rho^{2}=1 /(3-Y)$.

In most snub cases the quartic polynomial expression in (8) will factor and the relevant roots are found in a quadratic or cubic equation as summarized in Appendix A. For the notable snub subset $\mid 23 q$ or $\mid 2 \frac{3}{2} q$ the quartic polynomial in (8) will factor, leaving the relevant roots in the cubic equation

$$
Y^{3}-Y^{2}-Y+1-4 \cos ^{2} \frac{\pi}{q}=Y^{3}-Y^{2}-Y-1-2 \cos \frac{2 \pi}{q}=0 .
$$

Section 12 discusses how $Y$ is related to the characteristic facial twists of snub $\{3\}$ in | $23 q$. 


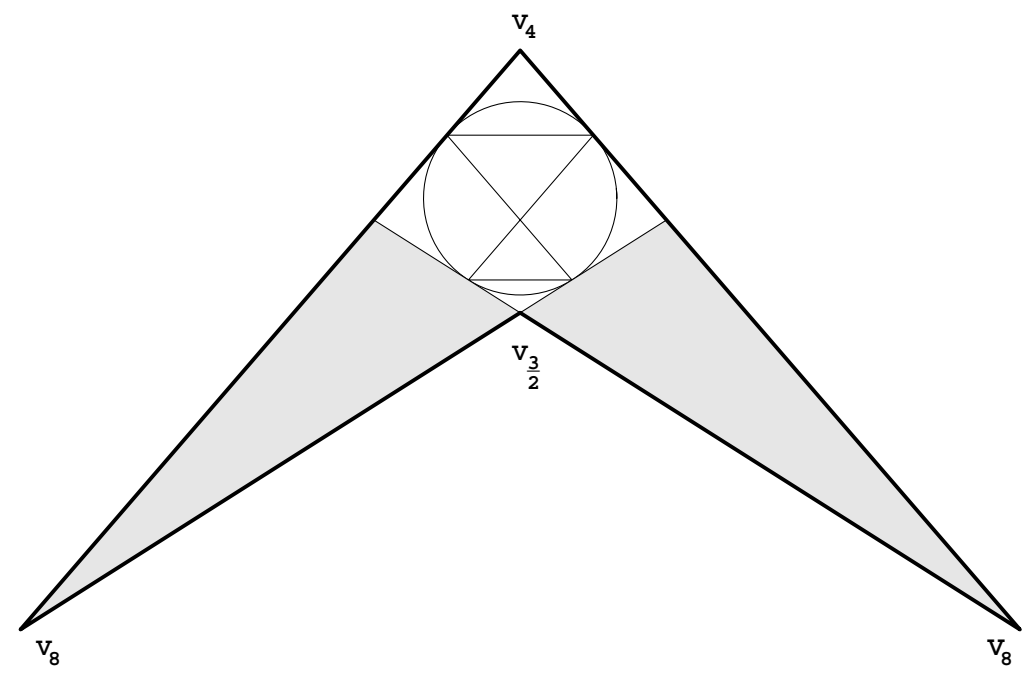

Fig. 3. Face of dual of $\frac{3}{2} 4 \mid 4$.

\section{Duals of Uniform Polyhedra}

Many of the previous terms and symbols used for uniform polyhedra are also used for the duals. A simple description of the dual (reciprocal) of a parent uniform polyhedron is a polyhedron that has the same number, $N_{1}$, of edges as the parent but there is an interchange in the number and placement of faces and vertices. Thus, an $\{n\}$ of the parent yields a regular $n$-gonal vertex $V_{n}$ of the dual polyhedron where $V_{n}$ lies on the ray $\vec{O}_{3} \mathrm{O}_{2}$. Moreover, the dual polyhedron has $N_{0}$ identical (or enantiomorphically paired) faces, each a $k$-sided polygon defined by the vertices $V_{n_{1}}, V_{n_{2}}, \ldots, V_{n_{k}}$ which follow the order noted in the parent vertex cycle $n_{1} \cdot n_{2} \cdots \cdot n_{k}$ described in Section 3. Duality implies that the circle which circumscribes the parent vertex figure also inscribes this dual $k$-gon. Therefore, the sides of the $k$-gon are simply the tangents constructed at the vertices of the parent vertex figure.

Figure 3 shows the dual face constructed around the vertex figure of $\frac{3}{2} 4 \mid 4$ which is also shown in Fig. 2 with labeled sides. Duals of non-convex uniform polyhedra have parts of their faces (often including some vertices) hidden from outside viewing. In Fig. 3 only the shaded portions of the dual face are visible on the surface of the complete dual polyhedron shown in Fig. 4. The corresponding parent uniform polyhedron $\frac{3}{2} 4 \mid 4$ is shown in Fig. 1 at a different scale and orientation. It turns out that the dual of

$$
24^{\frac{3}{2}}
$$

has the same outward appearance as Fig. 4 because each of its dual faces is exactly the shaded portions shown in Fig. 3. More of such apparent dual equivalencies and details for constructing all the duals can be found in [41]. 


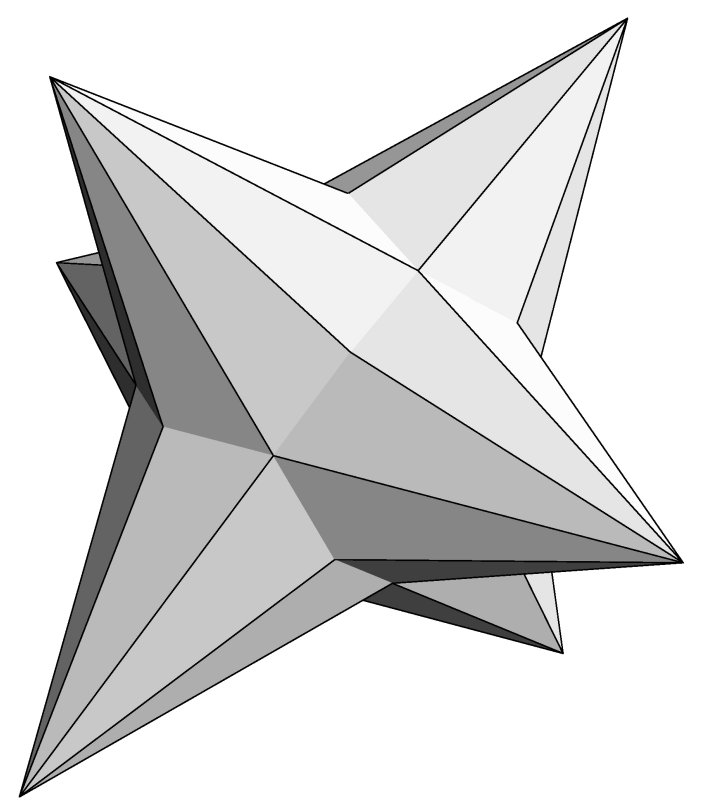

Fig. 4. Dual of $\frac{3}{2} 4 \mid 4$.

The following additional symbols are used for the duals:

$$
\begin{aligned}
\gamma_{n}= & \text { distance } V_{n} O_{3} . \\
\varepsilon_{n_{1}, n_{2}}= & \text { dual edge-length } V_{n_{1}} V_{n_{2}} . \\
\alpha_{n}= & \text { dual face angle at } V_{n} . \text { There are } \operatorname{num}(n) \text { equal dual face angles that emanate } \\
& \text { from } V_{n} . \\
{ }_{3} R= & \text { insphere radius of the dual polyhedron, distance from } O_{3} \text { to dual face. } \\
\delta^{\prime}= & \text { dihedral angle between two adjacent faces of the dual polyhedron. } \\
\rho= & \text { radius of the circle inscribed in the dual face (= circumradius of the parent } \\
& \text { vertex figure). }
\end{aligned}
$$

Metrical quantities here are specifically for the dual whose edges are tangent to the midsphere (radius ${ }_{1} R$ ) of the parent uniform polyhedron of edge-length 2 . Calculations are based on polar reciprocation with respect to this sphere [41]. Accordingly, these relations are generally known:

$$
\begin{gathered}
\delta^{\prime}+2 \phi=\pi ; \quad \sin \frac{\delta^{\prime}}{2}=\cos \phi=\rho ; \quad\left(\gamma_{n}\right)\left({ }_{2} R R_{n}\right)=\left({ }_{1} R\right)^{2}=\left({ }_{0} R\right)\left({ }_{3} R\right) \\
{ }_{3} R=\rho\left({ }_{1} R\right)=\rho^{2}\left({ }_{0} R\right) .
\end{gathered}
$$


The subsequent relations in this section are newly reported beginning with

$$
\begin{aligned}
\gamma_{n} & =\frac{\cot ^{2} \phi}{\sqrt{\cot ^{2} \phi-\cot ^{2}(\pi / n)}} ; \\
\varepsilon_{n_{1}, n_{2}} & =\frac{1}{\sqrt{\tan ^{2}\left(\pi / n_{1}\right)-\tan ^{2} \phi}}+\frac{1}{\sqrt{\tan ^{2}\left(\pi / n_{2}\right)-\tan ^{2} \phi}} ; \\
\cos \frac{\alpha_{n}}{2} & =\frac{\cos (\pi / n)}{\cos \phi} .
\end{aligned}
$$

Note that because face $\{2 r\}$ is equatorial $\left({ }_{2} R_{2 r}=0\right)$ in cases of $p q \mid r: 1 / p+1 / q=1$, we have $\phi=\pi / 2 r$ and so $\gamma_{2 r}$ becomes infinity.

We now briefly discuss some analytic geometry of dual polyhedra.

If $\left(x_{u}, y_{u}, z_{u}\right)$ is a vertex of the parent uniform polyhedron, then the reciprocally related center of the incircle of the dual face is $\left(\left({ }_{3} R /{ }_{0} R\right) x_{u},\left({ }_{3} R /{ }_{0} R\right) y_{u},\left({ }_{3} R /{ }_{0} R\right) z_{u}\right)=$ $\left(\rho^{2} x_{u}, \rho^{2} y_{u}, \rho^{2} z_{u}\right)$. The corresponding dual facial plane is represented by the equation in normal form $\left(x_{u} /{ }_{0} R\right) x+\left(y_{u} /{ }_{0} R\right) y+\left(z_{u} /{ }_{0} R\right) z-{ }_{3} R=0$ or in convenient form $\left(x_{u}\right) x+\left(y_{u}\right) y+\left(z_{u}\right) z={ }_{1} R^{2}=\cot ^{2} \phi$.

By using the scaling factor $1 / \rho^{2}={ }_{0} R /{ }_{3} R={ }_{1} R^{2} /{ }_{3} R^{2}={ }_{0} R^{2} /{ }_{1} R^{2}$, we expand the current dual whose edges are tangent to the midsphere to the larger dual whose faces are tangent to the parent's circumsphere (radius ${ }_{0} R$ ).

The equation of the corresponding facial plane of this expanded dual has the normal form $\left(x_{u} /{ }_{0} R\right) x+\left(y_{u} /{ }_{0} R\right) y+\left(z_{u} /{ }_{0} R\right) z-{ }_{0} R=0$ or the convenient form $\left(x_{u}\right) x+\left(y_{u}\right) y+$ $\left(z_{u}\right) z={ }_{0} R^{2}$.

The Cartesian coordinates of dual vertices $V_{p}, V_{q}, V_{r}$ are determined from each of $P, Q, R$ given for the parent uniform polyhedron in Section 5. For example, P: $\left(x_{p}, y_{p}, z_{p}\right)$ is unit distance from the origin and so we have the reciprocal $V_{p}:\left(\gamma_{p} x_{p}, \gamma_{p} y_{p}, \gamma_{p} z_{p}\right)$. $V_{p}$ is also $(k A, k B, k C)$ where $k=\gamma_{p} / \sqrt{A^{2}+B^{2}+C^{2}}$ and $A, B, C$ are coefficients in the general equation $A x+B y+C z+D=0$ of the parent facial plane $\{p\}$. It is generally known from analytic geometry that the equation of $\{p\}$ is determined from any of its three vertices $\left(x_{u}, y_{u}, z_{u}\right)$ by

$$
\left|\begin{array}{cccc}
x & y & z & 1 \\
x_{1} & y_{1} & z_{1} & 1 \\
x_{2} & y_{2} & z_{2} & 1 \\
x_{3} & y_{3} & z_{3} & 1
\end{array}\right|=A x+B y+C z+D=0 .
$$

\section{Some Simplified Formulas Unique to Regular Polyhedra}

Regular polyhedra are uniform polyhedra with one kind of face as evidenced by their Wythoff constructions $p \mid 2 q$ which yield vertex cycles $q^{p}$. They are the five convex Platonic solids and the four non-convex star polyhedra of Kepler-Poinsot, all included in the first group of Appendix B. A regular polyhedron, with face $\{p\}$ and regular $q$ gonal vertex figure, is conveniently denoted by the Schläfli symbol $\{p, q\}$. Note that 
$\{p, q\}$ corresponds to Wythoff symbol $q \mid p 2$ (or $q \mid 2 p$ ). For these cases we use $\chi=\chi_{p}, \psi=\psi_{p}$, and $\delta=\delta_{p, p}$.

The vertex for $\{p, q\}$ of edge-length 2 that corresponds to oriented $q \mid p 2$ or $q \mid 2 p$ is respectively

$$
\left(1,0,{ }_{1} R\right) \text { or }\left({ }_{0} r_{p}, 0,{ }_{2} R_{p}\right)
$$

where

$$
\begin{gathered}
{ }_{1} R^{2}=\cot ^{2} \phi=\left(\sec ^{2} \frac{\pi}{p} \sin ^{2} \frac{\pi}{q}-1\right)^{-1}, \quad{ }_{0} r_{p}=\csc \frac{\pi}{p}, \\
{ }_{2} R_{p}=\sqrt{\cot ^{2} \phi-\cot ^{2} \frac{\pi}{p}} .
\end{gathered}
$$

Interesting known relations [5] for $\{p, q\}$ are

$$
\begin{gathered}
\cos \phi=\cos \frac{\pi}{p} \csc \frac{\pi}{q} ; \quad \cos \chi=\cos \phi \cos \psi=\cos \phi \sin \frac{\delta}{2}=\cot \frac{\pi}{p} \cot \frac{\pi}{q} ; \\
\cos \psi=\sin \frac{\delta}{2}=\csc \frac{\pi}{p} \cos \frac{\pi}{q} .
\end{gathered}
$$

Expressions for $N_{0}, N_{1}$, and $N_{2}$ in terms of $p$ and $q$ are well known for the Platonic solids [5]:

$$
\begin{gathered}
N_{0}=\frac{4 p}{4-(p-2)(q-2)}, \quad N_{1}=\frac{2 p q}{4-(p-2)(q-2)}, \\
N_{2}=\frac{4 q}{4-(p-2)(q-2)} .
\end{gathered}
$$

\section{Relative Facial Twists and Edge Lengths of the Snub Polyhedron |pq 2: $\lambda=1$}

The family of snub polyhedra $\mid p q 2$ where $p>2, q>2$ is assigned the Schläfli symbol s $\left\{\begin{array}{l}p \\ q\end{array}\right\}$. In several references [2], [9], [17], [19], [20], [30], [32] we find examples of the convex snub polyhedra s $\left\{\begin{array}{l}3 \\ 3\end{array}\right\}, \mathrm{s}\left\{\begin{array}{l}3 \\ 4\end{array}\right\}, \mathrm{s}\left\{\begin{array}{l}3 \\ 5\end{array}\right\}$ encased by the proper $\{p, q\}$ or $\{q, p\}$ in order to observe the rotation angle of snub faces $s\{p\}$ or $s\{q\}$ relative to the coplanar Platonic faces $\{p\}$ or $\{q\}$. The specific associations for the convex cases are: $\mathrm{s}\{3\}$ relates to $\{3,3\},\{3,4\}$, or $\{3,5\} ; \mathrm{s}\{4\}$ relates to $\{4,3\} ; \mathrm{s}\{5\}$ relates to $\{5,3\}$. The regular icosahedron $\{3,5\}$ can be interpreted as $\mathrm{s}\left\{\begin{array}{l}3 \\ 3\end{array}\right\}$ or $\mid 233$ which is encased in the regular tetrahedron $\{3,3\}$.

In this section we extend the encasing relationship to include non-convex snub cases and retrograde cases $\mid p q 2$ where $p<2$ and/or $q<2$. Facial twist $\theta_{p}$ is defined as the smallest angle between a symmetry line locally within $\mathrm{s}\{p\}$ and a symmetry 
plane member of the full symmetry group that is superposed on the rotational group of $\mid p q$ 2. It follows that $0 \leq \theta_{p}<\pi / 2 \operatorname{num}(p)$ and specifically $\theta_{p}=0$ for the reflexible case | $p q r$ where $q=r$. Using Wythoff symbols as subscripts we generalize to $\cot ^{2} \phi_{q \mid p 2}=\left(\sec ^{2}(\pi / p) \sin ^{2}(\pi / q)-1\right)^{-1}$ from the relations in (10) used for the regular polyhedron $q \mid p 2$. Then $\theta_{p}$ of $\mid p q 2$ is the least angle of the set $|k \pi / \operatorname{num}(p)-\mu|$ where $k=0,1,2, \ldots, \operatorname{num}(p)-1$ and

$$
\begin{aligned}
& \sin \frac{\pi}{p}\left(\cot \phi_{q \mid p 2} \cot \phi_{\mid p q 2}+\operatorname{sgn}(2-q)\right. \\
& \mu=\arccos \frac{\times \sqrt{\left.\left(\cot ^{2} \phi_{q \mid p 2}-\cot ^{2} \frac{\pi}{p}\right)\left(\cot ^{2} \phi_{\mid p q 2}-\cot ^{2} \frac{\pi}{p}\right)\right)}}{\cot \frac{\pi}{p}} .
\end{aligned}
$$

For the non-retrograde case $\mid p q 2$ where $p>2$ and $q>2$, we note that $k=1$ for example.

If we now assign $p=3$, that is $\mid 3 q 2$ where $q>2$ or $q<2$, a simpler expression for $\theta_{3}$ is

$$
\xi=\frac{\pi}{6}-\arcsin \frac{1}{2} \sqrt{\frac{3 \cot ^{2} \phi_{q \mid p 2}-1}{3 \cot ^{2} \phi_{\mid p q 2}-1}} .
$$

If $p=\frac{3}{2}$ as in $\mid \frac{3}{2} q 2$, then we must adjust to $\theta_{3 / 2}=\pi / 3+\xi$.

It has been shown in [17] that for the small family s $\left\{\begin{array}{l}3 \\ q\end{array}\right\}$ or $\mid 23 q$ where $q>2$, each extended side of the twisted $\mathrm{s}\{3\}$ intersects a vertex and a side of the encasing $\{3\}$ of $\{3, q\}$. The lesser of the two angles that an extended side of $s\{3\}$ makes with the sides of $\{3\}$ is the same measurement as our defined $\theta_{p}$ for $p=3$. In terms of $\theta_{3}$, we find by elementary geometry that the sides of $\{3\}$ are cut in the ratio $Y=\left(\sqrt{3} \cot \theta_{3}-1\right) / 2$. Rotge [30] demonstrated that $Y^{3}-Y^{2}-Y-1+2 \cos A=0$ where $A$ is the (interior) vertex angle in the face $\{q\}$. However, $A=\pi(1-2 / q)$ and so we have $Y^{3}-Y^{2}-Y-1-2 \cos (2 \pi / q)=$ 0 which is the same as (9) in variable $Y=2-\tan ^{2} \phi$ that is associated with the snub subset $\mid 23 q$ in Section 9. From $\cot \theta_{3}=(1+2 Y) / \sqrt{3}$, we obtain the compact relation $2 \tan ^{2} \phi+\sqrt{3} \cot \theta_{3}=5$ for $\mid 23 q$ where $q>2$.

For the retrograde case $\mid 23 \frac{5}{3}$ we must select the greater complementary twist angle $\pi / 3-\theta_{3}$ so that $Y=(\sqrt{3} / 2) \cot \left(\pi / 3-\theta_{3}\right)-\frac{1}{2}$ satisfies (9).

Identifying edges and insphere radii with Wythoff symbol subscripts, we calculate the ratio of edge lengths when $s\{p\}$ lies in $\{p\}$ of $q \mid p 2$ from

$$
\frac{e_{\mid p q 2}}{e_{q \mid p 2}}=\frac{{ }_{2} R_{p(q \mid p 2)}}{{ }_{2} R_{p(\mid p q 2)}}=\sqrt{\frac{\cot ^{2} \phi_{q \mid p 2}-\cot ^{2}(\pi / p)}{\cot ^{2} \phi_{\mid p q 2}-\cot ^{2}(\pi / p)}} .
$$

The subscripts in (11) could actually be changed to any two Wythoff symbols when $p$-gon facial planes are shared by two uniform polyhedra.

In the next section facial twists for the snub cases other than $\mid p q 2$ are solved by a general analytic geometry approach. 


\section{Relative Facial Twists for Any Snub Polyhedron $\mid p q r$}

The Cartesian orientation of snub polyhedron | $p q r$ as described in Section 5 specifically places $s\{r\}$ centrally and orthogonally about the $Z$-axis. Thus, the facial twist $\theta_{r}$ is the least angle of the set $\left|k \pi / \operatorname{num}(r)-\arctan \left(y_{w} / x_{w}\right)\right|$ where $k=0,1,2$ and

$$
\frac{y_{w}}{x_{w}}=\frac{\sin (\pi / r)}{w+\cos (\pi / r)} .
$$

The coordinates $x_{w}$ and $y_{w}$ of the snub vertex in (5) are calculated by solving quartic equation (4) in the variable $w$ in Section 6. As previously stated, specific cases | $33 \frac{5}{2}$ and $\mid \frac{3}{2} \frac{3}{2} \frac{5}{2}$ are reflexible because two Wythoff elements are identical. Here we find non-twisted $s\left\{\frac{5}{2}\right\}$ placed symmetrically about the reflection planes unlike $s\{3\}$ and $s\left\{\frac{3}{2}\right\}$ which are twisted. Similarly, $\{p\}$ of antiprism $\mid p 22$ is not twisted. All facial twists for | $p q r: \lambda=1$ are summarized in Appendix A.

\section{The Infinite Families of Uniform Prisms and Antiprisms}

Although the formulas in previous sections apply to prisms $(p 2 \mid 2: p>2$ yielding $p \cdot 4 \cdot 4)$, antiprisms $\left(\mid p 22: p>\frac{3}{2}\right.$ and $p \neq 2$ yielding $\left.p \cdot 3 \cdot 3 \cdot 3\right)$, and their duals, a few convenient simplifications for edge-length 2 are tabulated here. Antiprisms can be separated into "ordinary antiprisms" $(p>2)$ and "crossed antiprisms" $\left(\frac{3}{2}<p<2\right)$ which have crossed vertex figures.

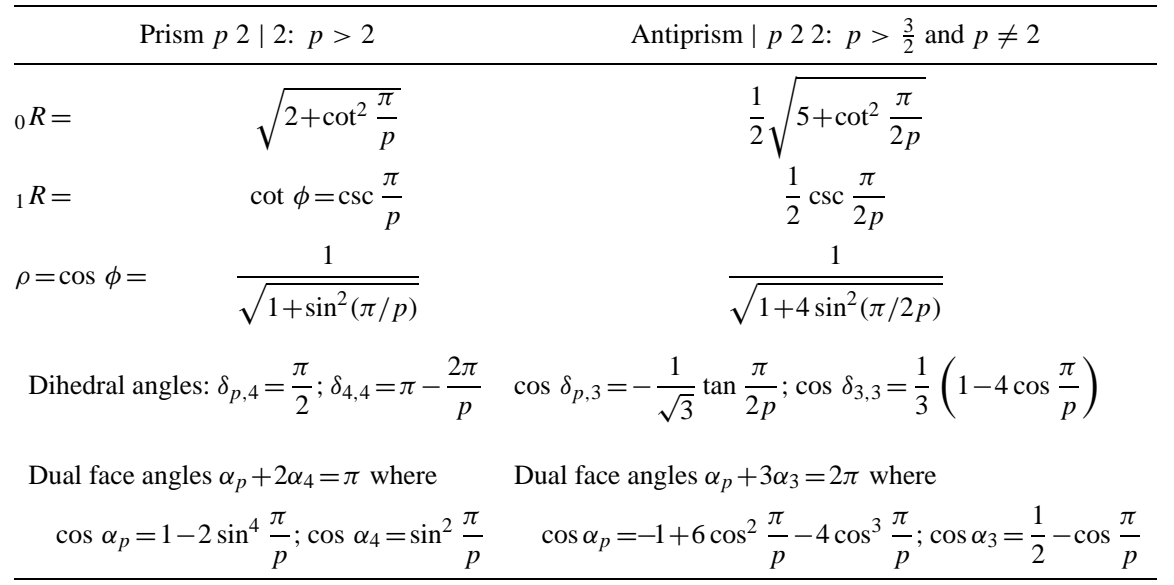

\section{Acknowledgment}

The author expresses gratitude to H. S. M. Coxeter, Branko Grünbaum, and Norman W. Johnson for their helpful comments. 


\section{Appendix A. Essential Equations and Angles for Uniform Snub Polyhedra $\mid p q r$}

Each uniform snub polyhedron has a fourth degree "snub equation" (8) in the variable $Y=2-\tan ^{2} \phi$ as described in Section 9. A half-edge subtends angle $\phi$ at the center $\mathrm{O}_{3}$ of the polyhedron. In most cases the quartic polynomial expression in (8) will factor and the relevant roots are found in a quadratic or cubic equation as shown below. For example, cases $\mid 33 \frac{5}{2}$ and $\mid \frac{3}{2} \frac{3}{2} \frac{5}{2}$ relate to $Y^{2}-Y-\tau=0$ where golden ratio $\tau=(1+\sqrt{5}) / 2=1.6180339887$ and so $Y=(1 \pm \sqrt{1+4 \tau}) / 2$. Decimal results are truncated, not rounded. Facial twist $\theta_{n}$ is the (smallest) angle that the snub face $\mathrm{s}\{n\}$ is rotated relative to the encasing face $\{n\}$ of the fully symmetric polyhedron that encases the snub polyhedron. For the family | $23 q$ where $q>2$ we have $\cot \theta_{3}=$ $(1+2 Y) / \sqrt{3}$ and $2 \tan ^{2} \phi+\sqrt{3} \cot \theta_{3}=5$. Special cases $\mid 233$ and $\mid 2 \frac{3}{2} \frac{3}{2}$ are equivalent to the Platonic icosahedron $5 \mid 23$ and the regular star icosahedron $\frac{5}{2} \mid 23$, respectively.

\begin{tabular}{|c|c|c|c|c|c|}
\hline $\mid p q r$ & Snub equation & $Y=2-\tan ^{2} \phi$ & $\phi$ (degrees) & \multicolumn{2}{|c|}{ Facial twist $\theta_{n}$ for $s\{n\}$ (degrees) } \\
\hline $\begin{array}{l}1233 \\
12 \frac{3}{2} \frac{3}{2} \\
\end{array}$ & $Y^{2}\left(Y^{2}-Y-1\right)=0$ & $\begin{array}{c}\tau \\
-\tau^{-1} \\
\end{array}$ & $\begin{array}{l}31.717474411 \\
58.282525588 \\
\end{array}$ & $\begin{array}{c}\theta_{3}=22.238756092 \\
\theta_{3 / 2}=22.238756092\end{array}$ & \\
\hline 234 & $\begin{array}{l}(Y-1) \\
\cdot\left(Y^{3}-Y^{2}-Y-1\right)=0\end{array}$ & 1.8392867552 & 21.845383553 & $\theta_{3}=20.315014336$ & $\theta_{4}=16.467560400$ \\
\hline 235 & $\begin{array}{l}(Y-\tau) \\
\cdot\left(Y^{3}-Y^{2}-Y-\tau\right)=0\end{array}$ & 1.9431512592 & 13.410633720 & $\theta_{3}=19.517922567$ & $\theta_{5}=13.106403376$ \\
\hline $23 \frac{5}{2}$ & & 1.3990206456 & 37.783865694 & $\theta_{3}=24.514783895$ & $\theta_{5 / 2}=8.8920818930$ \\
\hline $23 \frac{5}{3}$ & $\begin{array}{l}\left(Y+\tau^{-1}\right) \\
\left(Y^{3}-Y^{2}-Y+\tau^{-1}\right)=0\end{array}$ & 0.4944394214 & 50.820400469 & $\theta_{3}=18.948438216$ & $\theta_{5 / 3}=10.155303612$ \\
\hline $12 \frac{3}{2} \frac{5}{3}$ & & -0.8934600671 & 59.549433143 & $\theta_{3 / 2}=5.5663456794$ & $\theta_{5 / 3}=16.952614494$ \\
\hline $\begin{array}{l}25 \frac{5}{2} \\
25 \frac{5}{3} \\
\end{array}$ & $Y^{4}-Y^{3}-3 Y^{2}+5=0$ & $\begin{array}{l}1.8180755760 \\
1.4739876869 \\
\end{array}$ & $\begin{array}{l}23.099507883 \\
35.952140323 \\
\end{array}$ & $\begin{array}{l}\theta_{5}=14.519275160 \\
\theta_{5}=10.766941734\end{array}$ & $\begin{array}{l}\theta_{5 / 2}=12.068113640 \\
\theta_{5 / 3}=7.6119024894\end{array}$ \\
\hline $\begin{array}{l}133 \frac{5}{2} \\
1 \frac{3}{2} \frac{3}{2} \frac{5}{2} \\
\end{array}$ & $\begin{array}{l}\left(Y+\tau^{-1}\right)^{2} \\
\left(Y^{2}-Y-\tau\right)=0\end{array}$ & -0.8667603991 & 59.433273863 & $\begin{array}{c}\theta_{3}=27.640276588 \\
\theta_{3 / 2}=27.640276588\end{array}$ & $\begin{array}{l}\theta_{5 / 2}=0 \\
\theta_{5 / 2}=0\end{array}$ \\
\hline $35 \frac{5}{3}$ & $\begin{array}{l}(2 Y-3) \\
\cdot\left(Y^{3}-2 Y^{2}+Y-1\right)=0\end{array}$ & 1.7548776662 & 26.339933901 & $\begin{array}{c}\theta_{3}=10.518676134 \\
\theta_{5 / 3}=15.401415840\end{array}$ & $\theta_{5}=7.1174491250$ \\
\hline | $3 \frac{5}{2} \frac{5}{3}$ & $\begin{array}{l}\left(\sqrt{5} Y-\tau^{-3}\right)\left(Y+\tau^{-1}\right) \\
\cdot(Y-1)^{2}=0\end{array}$ & 1 & 45 & $\begin{array}{c}\theta_{3}=14.330332524 \\
\theta_{5 / 3}=15.772242400\end{array}$ & $\theta_{5 / 2}=15.772242400$ \\
\hline
\end{tabular}

\section{Appendix B. The 77 Wythoff Symbols and Corresponding Vertex Cycle Symbols}

Wythoff symbols for the uniform polyhedra are grouped by type and listed in columns according to the increasing numerical order of numerators and denominators. Vertex 
cycles preserve the symbols used for retrograde faces. See Section 3 for an explanation of Coxeter's "hybridized" Wythoff symbol used for $p q r \mid$ having an even denominator.

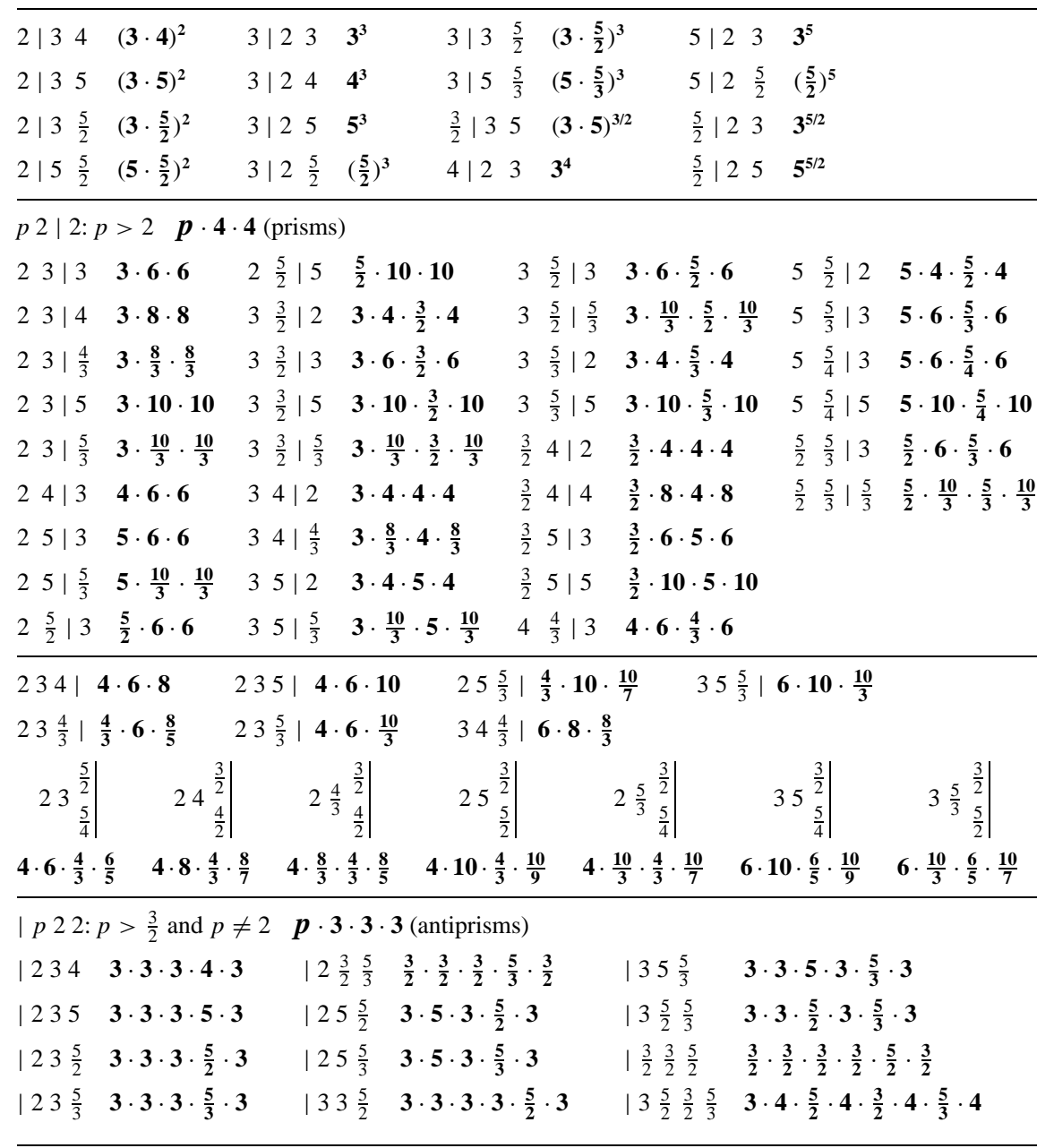

\section{Bibliography}

To various degrees the following references provide formulas, quantities, and images for either the full set or subsets of uniform polyhedra and duals.

1. Ball, W. W. R., Coxeter, H. S. M. Mathematical Recreations and Essays, 13th edn, Dover, New York, 1987.

2. Beard, Col. R. S. Patterns in Space. Creative Publications, Palo Alto, CA, 1973.

3. Bulatov, V. Virtual models of uniform polyhedra and duals at http://www.physics.orst.edu/ bulatov/ polyhedra/.

4. Coxeter, H. S. M. Introduction to Geometry, 2nd edn., Wiley, New York, 1969.

5. Coxeter, H. S. M. Regular Polytopes, 3rd edn., Dover, New York, 1973. 
6. Coxeter, H. S. M. Star polytopes and the Schläfli function $f(\alpha, \beta, \gamma)$. Elemente der Mathematik, $\mathbf{4 4}(2)$ (1989), 25-36.

7. Coxeter, H. S. M., Longuet-Higgins, M. S., Miller, J. C. P. Uniform polyhedra. Philosophical Transactions of the Royal Society of London Series A, 246 (1954), 401-450.

8. Critchlow, K. Order in Space: A Design Source Book. Viking Press, New York, 1970.

9. Cundy, H. M. Snubbing with and without eta. The Mathematical Gazette, 84(499) (2000), 14-23.

10. Cundy, H. M., Rollett, A. P. Mathematical Models, 3rd. edn. Tarquin, Norfolk, 1981.

11. Gabriel, J. F. (editor). Beyond the Cube: The Architecture of Space Frames and Polyhedra. Wiley, New York, 1997.

12. Gasson, P. C. Geometry of Spatial Forms. Wiley, New York, 1983.

13. Graziotti, U. A. Polyhedra: The Realm of Geometric Beauty. University of San Francisco, San Francisco, CA, 1962.

14. Har'El, Z. Uniform solution for uniform polyhedra. Geometriae Dedicata, 47 (1993), 57-110.

15. Holden, A. Shapes, Space, and Symmetry. Columbia University Press, New York, 1971. Dover, New York, 1991.

16. Hume, A. Exact descriptions of regular and semi-regular polyhedra and their duals. Computing Science Technical Report No. 130, AT\&T Bell Laboratories, 1986.

17. Huybers, P., Coxeter, H. S. M. A new approach to the chiral Archimedean solids. Comptes Rendus Mathématiques, L'Academie des Sciences, Canada, 1(5) (1979), 269-274.

18. Johnson, N. W. Uniform Polytopes. Cambridge University Press, Cambridge (forthcoming).

19. Kappraff, J. Connections: The Geometric Bridge Between Art and Science, 2nd edn. World Scientific, Singapore, 2001.

20. Lines, L. Solid Geometry. Dover, New York, 1965.

21. Maeder, R. E. Uniform polyhedra. The Mathematica Journal, 3(4) (1993), 48-57. The electronic supplement contains the Mathematica program UniformPolyhedra.m which renders the uniform polyhedra; also available at http://www.mathsource.com/. Reprinted in The Mathematica Programmer II, Academic Press, New York, 1996.

22. Messer, P. W. Problem \#1094 (dihedral angle). Crux Mathematicorum, 11(10) (1985), 325. Solution\#1094. Crux Mathematicorum, 13(4) (1987), 133.

23. Messer, P. W. Stellations of the rhombic triacontahedron and beyond. Structural Topology, 21 (1995), $25-46$.

24. Pearce, P. \& S. Polyhedra Primer. Van Nostrand Reinhold, New York, 1978.

25. Peck, C. E. A Taxonomy of Fundamental Polyhedra and Tessellations. Winkworks, Wichita, KS, 1995.

26. Procházka, J. Polyèdres (French). Laval University Press, Quebec, 1975.

27. Pugh, A. Polyhedra: A Visual Approach. University of California Press, Berkeley, CA, 1976.

28. Roaf, D. Angles in Platonic solids. Mathematical Spectrum, 25(2) (1992/93), 47-51.

29. Roman, T. Reguläre und halbreguläre polyeder (German). VEB Deutscher Verlag der Wissenschaften, Berlin, 1968. Translated from original Romanian title Poliedre regulate si semiregulate (1965).

30. Rotge, J. F. Rotating polyhedra with congruent plane pentagonal faces. Structural Topology, 9 (1984), $15-30$.

31. Senechal, M., Fleck, G. (editors). Shaping Space: A Polyhedral Approach. Birkhäuser, Boston, 1988.

32. Sharp, J. Have you seen this number. The Mathematical Gazette, 82(494) (1998), 203-214.

33. Skilling, J. The complete set of uniform polyhedra. Philosophical Transactions of the Royal Society of London, Series A, 278 (1975), 111-135.

34. Sopov, S. P. A proof of the completeness of the list of elementary homogeneous polyhedra (Russian). Ukrainskii Geometricheskii Sbornik, 8 (1970), 139-156.

35. Szepesvári, I. Az uniform poliédereck számáról I.-II. (Hungarian). Matematikai Lapok, 29(4) (1981), 273-328.

36. Todhunter, I., Leathem, J. G. Spherical Trigonometry. Macmillan, New York, 1932.

37. Verheyen, H. F. Dipolygonids, Mobile Generators of Uniform Polyhedra. University of Antwerp, preprint series UIA (1979), 79-40. 2nd edn. (1981) retitled An Alternative Generation and Transformation Theory for Uniform Polyhedra.

38. Weisstein, E. W. CRC Concise Encyclopedia of Mathematics. CRC Press, Boca Raton, FL, 1999. CD-ROM version, 1999. An expanded Internet version, MathWorld at http//:www.wolfram.com/, contains links to 
the Mathematica programs UniformPolyhedra.m and Duals.m which render the uniform polyhedra and duals.

39. Wenninger, M. J. Polyhedron Models. Cambridge University Press, Cambridge, 1971.

40. Wenninger, M. J. Spherical Models. Cambridge University Press, Cambridge, 1979. Dover, New York, revised 1999.

41. Wenninger, M. J. Dual Models. Cambridge University Press, Cambridge, 1984.

42. Williams, R. The Geometrical Foundation of Natural Structure. Dover, New York, 1979.

Received September 20, 2000, and in revised form September 20, 2001. Online publication February 19, 2002. 